\title{
Nanoscale mechanism of photo-induced metastability and reversible photodarkening in chalcogenide vitreous semiconductors
}

\author{
(C) A.V. Kolobov ${ }^{\dagger, *}$, K. Tanaka \\ $\dagger$ Joint Research Center for Atom Technology - National Institute for Advanced Interdisciplinary Research, \\ 1-1-4 Higashi, Tsukaba, Ibaraki 305, Japan \\ * A.F. loffe Physicotechnical Institute, Russian Academy of Sciences, \\ 194021 St.Petersburg, Russia
}

(Получена 28 января 1998 г. Принята к печати 23 февраля 1998 г.)

The present paper reviews the results oblained by the authors in the last three years. By applying in-situ (pamp and probe) EXAFS to reversible photostructural changes in chalcogenide vitreous semiconductors we have found an increase in the average coordination number of selenium species in the photoexcited state which comes from the formation of dynamic interchain bonds. Subsequent bond rupture, studied by optically-induced ESR, results in the formation of over- and undercoordinated ESR-active defects which are further transformed into charged valencealternation pairs. Annealing close to the glass-transition temperature results in recovery of the initial structure. Relationship between the detected structural changes and photodarkening is also discussed.

\section{Introduction}

Being intrinsically non-equilibrium materials, amorphous semiconductors undergo a number of metastable transformations under the action of photo-excitaion, typical examples being Staebler-Wronski effect in hydrogenated amorphous silicon $(a-\mathrm{Si}: \mathrm{H})$ and reversible photostructural changes, also known as reversible photodarkening, in chalcogenide glasses. For recent reviews see [1-5].

Reversible photodarkening has been known since the early $70 \mathrm{~s}$ and manifested itself in a reversible shift of the absorption edge to lower energies under irradiation with the bandgap light $[6,7]$. X-ray diffraction studies have demonstrated that the photodarkening is closely related with changes in the structure [8], after which the terms "photodarkening" and "photostructural changes" have been used interchangeably.

Phenomenological explanation of the effect was given within the configuration-coordinate model which assumed double-well adiabatic potential for the ground state of the system with light transfering the system to the higher-energy, metastable, state [9-12]. Annealing returns the system to the ground state. This approach, being quite successful in describing the phenomenon could not, obviously, give any information regarding the nanoscale mechanism of the process.

Ranam scattering and EXAFS (extended X-ray absorption fine structure) studies have shown that the number of socalled "wrong bonds" (such as As-As bonds in stoichiometric $\mathrm{As}_{2} \mathrm{~S}_{3}$ glass which ideally should contain only As-S bonds) increased reversibly under irradiation $[13,14]$. This result, together with the finding that the photodarkening increased strongly in As-rich glasses [15], suggested a special role of arsenic in the process. An attempt to find such structural changes in elemental amorphous arsenic has, however, failed [16]. At the same time such an effect was clearly observed in elemental amorphous selenium $(a$-Se) [17-19] which made it clear that presence of chalcogen is crucial for the photostructural changes to take place.

Most of the studies were performed ex-situ when the sample before and after irradiation was studied. It is clear that under such conditions, at best, what happens as a result of the photoexcitation could be detected but not how it happens. We have undertook in-situ EXAFS, Raman and ESR measurements on elemental $a$-Se and we have been able to detect several stages of the photostructural changes, the description of which makes the subject of this paper.

\section{Experimental}

The samples have been prepared by thermal evaporation of bulk material onto silica-glass or aluminium-foil substrates held at room temperature in a vacuum. The film thickness was $\sim 1000 \AA$ for the EXAFS and Raman studies and a few microns for the ESR measurements.

EXAFS experiments have been performed in the fluorescence mode at beamline 13B at the Photon Factory. Detailed description of the experimental procedure is given elswhere [20]. Raman scattering was measured in the backscattering geometry and is described in more detail in ref. [21]. Experimental details of the ESR measurement can be found in ref. [22].

\section{Results and discussion}

\subsection{Photo-excited state}

Fig. 1, $a$ presents raw EXAFS oscillations measured at $30 \mathrm{~K}$ for an annealed film of $a$-Se and for the photo-excited $a$-Se as a function of wavenumber. One can clearly see that the amplitude of oscillations increases in the photoexcited state. Detailed data analysis based on the single scattering theory [23] and curve-fitting as well as the socalled ratio-method [24] have revealed that in the photo- 
excited state the average coordination number of selenium species increases (Fig. 2) dynamically acquiring its original value after turning off the exciting light. This increase in coordination is believed to be caused by the following process.

Under the photo-excitation, one of the lone-pair (LP) electrons which form the top of the valence band is excited, while the other one is left unpaired in the former LP-orbital. Provided the time of the carrier localization is long enough, the unpaired LP electron interacts with the neighbouring chain and forms an interchain bond increasing the local coordination of selenium atoms. Once the third bond is formed, with two electrons lowering their energy from the non-bonding state to the bonding state, the corresponding empty states move to the anti-binding state leaving no empty state behind. For this reason, recombination of the photo-excited electrons is impossible in the three-fold coordinated geometry and bond rupture is a necessary condition for the recombination, which means that the newly formed bonds are essentially dynamic. It sould be beared in mind that, once formed, the new bonds are
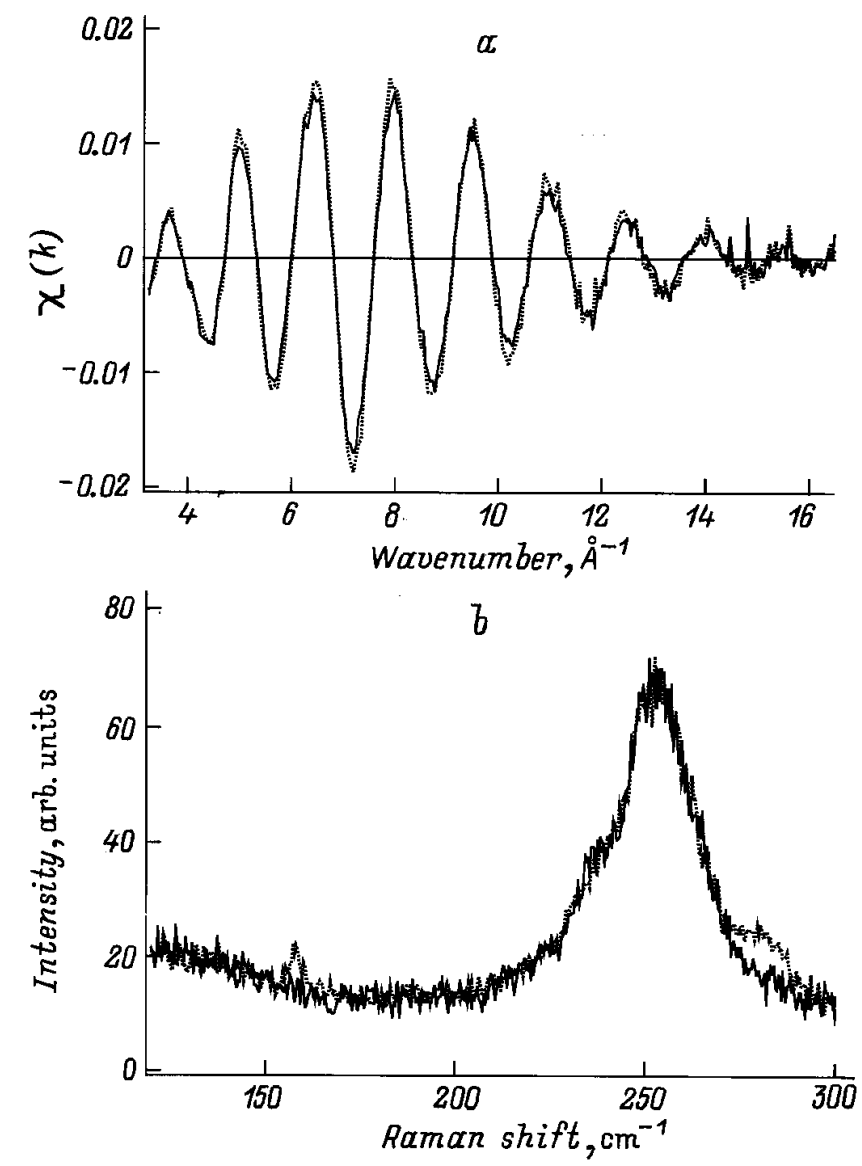

Figure 1. Raw EXAFS oscillations measured at $30 \mathrm{~K}$ as a function of wavenumber $(a)$ and Raman spectra at the same temperature $(b)$ for the annealed (solid line) and photo-excited (dotted line) $a$-Se film. An increase in the amplitude of the EXAFS oscillation and appearence of new peaks in Raman spectra in the photo-excited state are clearly seen.

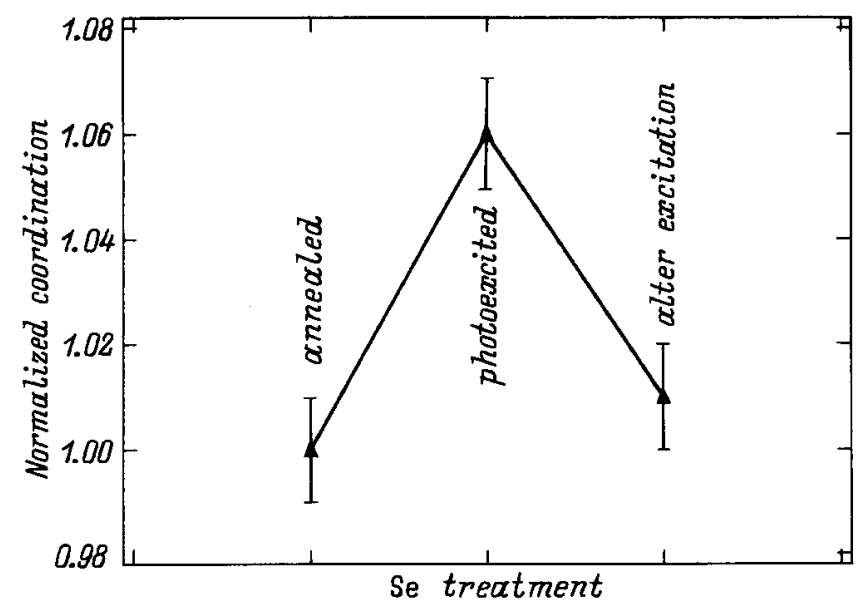

Figure 2. Change in the average coordination number of $a$-Se at various stages of the film treatment. A reversible increase in the coordination appears in the photo-excited state.

identical to the "old" bonds subtended at the same overcoordinated atoms, which means the system possesses pairs of overcoordinated atoms dynamically incorporated into the rest of the matrix. Structure relaxation and bond rupture in these nanovolumes results in a metastable reversible change and explains recently observed photo-induced fluidity of chalcogenide glasses [25]. Our data demonstrate that the concentration of such pairs reaches $7 \%$.

In order to confirm the conclusion made on the basis of the EXAFS experiment, an in-situ Raman scattering study of the photostructural change in $a$-Se has been performed [21]. Fig. 1, $b$ shows polarized Raman spectra ( $H V$-configuration) measured at $20 \mathrm{~K}$ for annealed and photo-excited films of $a$-Se. Two peaks, previously not reported, are clearly seen in the photo-excited state at 158 and $280 \mathrm{~cm}^{-1}$. Although anambiguous assignment of these peaks is not straightforward they clearly demonstrate that bonding in the photo-excited state is changed and can be readily explained by the formation of the interchain bonds. Indeed, the formation of the third bond makes the structure more rigid and should result in an increase in the frequency (the peak located at $280 \mathrm{~cm}^{-1}$ ). Neutron scattering study of liquid selenium, which is argued to possess around $15 \%$ of threefold coordinated atoms [26,27], has also shown a similar high-frequence shift [28]. The peak at $158 \mathrm{~cm}^{-1}$ possibly results from a shift of the $150 \mathrm{~cm}^{-1}$ peak due to $E^{\prime}$ mode to higer frequencies because of increased interchain interaction in the cross-linked geometry in the photo-excited state.

\subsection{Bond reconstruction following the photo-excitation}

Rupture of dynamic bonds following the photo-excitation should result in creation of unpaired electrons and hence an ESR signal which is not observed in annealed chalcogenide glasses [29]. Indeed, we were able to detect a strong photoinduced ESR signal in our samples (Fig. 3). With annealing 


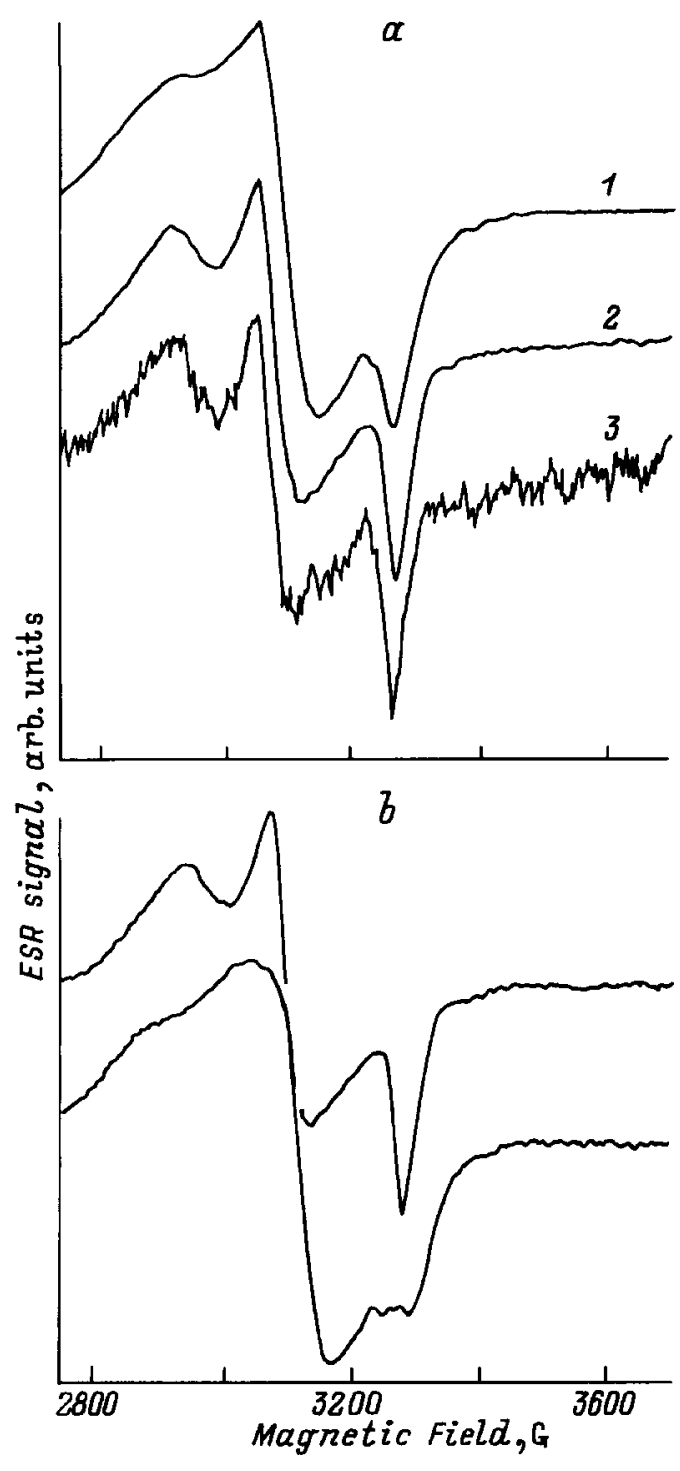

Figure 3. $a-$ photo-induced ESR signal and its temperature evolution; annealing temperatrue is $20(1), 90(2), 150(3)^{\circ} \mathrm{C}$; all spectra were measured at $20 \mathrm{~K} . b$ - anisotropic (top) and isotropic (bottom) components of the ESR signal.

(measurements were always done at $20 \mathrm{~K}$ ) the lineshape of the signal changed in the temperature range from 20 to $90 \mathrm{~K}$ suggesting the presence of more than one center (Fig. 3,a) while annealing at higher temperatures resulted only in a desrease of the signal intensity. After annealing at $160 \mathrm{~K}$ ESR signal desappears. Deconvolution of the spectra into two components is shown in Fig. 3, $b$. The more stable component is anisotropic and characterised by $g$-factros $g_{1}=2.22, g_{2}=2.099$ and $g_{3}=1.983$. The other one is isotropic and has $g$-factor value of 2.09 .

The obtained $g$-factor values for the anisotropic center agree very well with the calculated values $\left(g_{1}=2.22\right.$, $g_{2}=2.075$ and $g_{3}=2.0023$ [30]) for the neutral chain end (singly coordinated defect). We believe that the other one comes from a three-fold coordinated defect. The observed density of the photo-induced spins $\left(\sim 10^{20} \mathrm{~cm}^{-3}\right)$ agrees well with the concentration of the three-fold coordinated pairs detected in the EXAFS experiment.

Fig. 4 shows the temperature dependence of partial concentrations of the two types of ESR-active centers. It is seen that the concentration of three-fold coordinated defects gradually decreases with the temperature increase and disappears at around $90 \mathrm{~K}$. Interestingly, the concentration of the other species increases in the same temperature range demonstrating that there is a conversion of threefold coordinated defects into singly coordinated ones. This experiment is in contrast with simple considerations of the valence-alternation pair (VAP) model [31] but is in full agreement with the results of later calculations [32]. Our EXAFS measurements during the annealing of the irradiated sample in the range from 20 to $160 \mathrm{~K}$ (not shown, see [33]) provide further evidence for the change in the defect coordination number upon annealing.

At temperatures higher than $160 \mathrm{~K}$ the photo-induced ESR signal disappears but if the irradiation is performed again at low temperature, the built-up of the signal proceeds much faster than in the primary irradiation cycle. This result is similar to that reported for binary $\mathrm{As}_{2} \mathrm{~S}_{3}$ glass [34] and implies that the photo-induced defects are not annealed out of $160 \mathrm{~K}$, only their charge state is changed at that temperature. Subsequent irradiation at low temperature does not require creation of defects any more but just their excitation which proceeds at a much faster rate. We have argued $[22,23]$ that at temeratures above $160 \mathrm{~K}$ charged valence-alternation pairs $\left(\mathrm{C}_{3}^{+} \mathrm{C}_{1}^{-}\right)$are formed.

Annealing at temperatures close to room temperature (the glass-transition temperature for $a$-Se is around $30^{\circ} \mathrm{C}$ ) results in complete recovery of the sample initial parameters [17].

The complete structural change taking place in $a$-Se consists thus in the following (Fig. 5). Under photo-excitation, lone-pair electrons are excited and dynamic interchain bonds

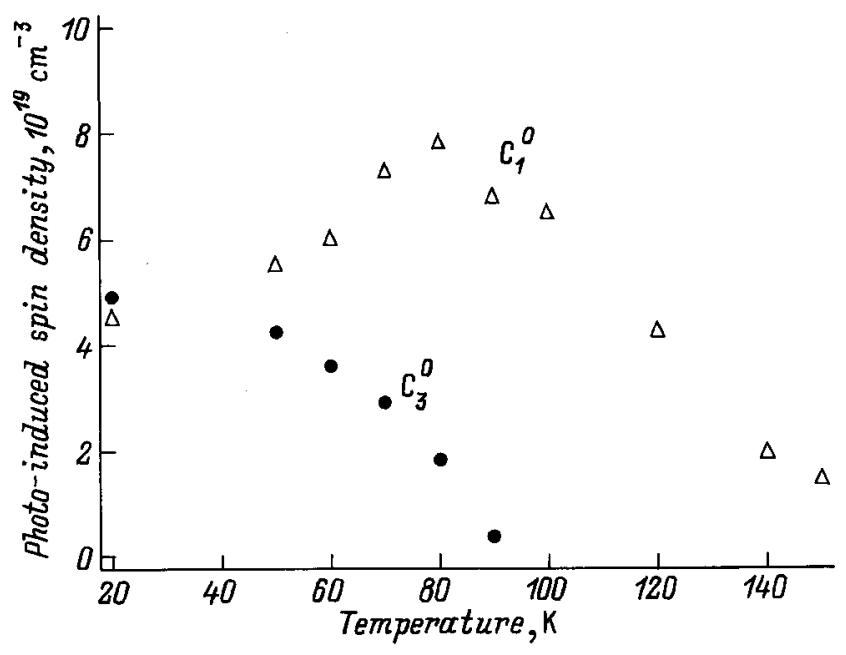

Figure 4. Temperature dependencies of partial concentration of the two kinds of ESR-active centers. Three-fold coordinated centers $\left(\mathrm{C}_{3}^{\circ}\right)$ are transformed into single coordinated ones $\left(\mathrm{C}_{1}^{\circ}\right)$ in the temperature range from 20 to $90 \mathrm{~K}$. 


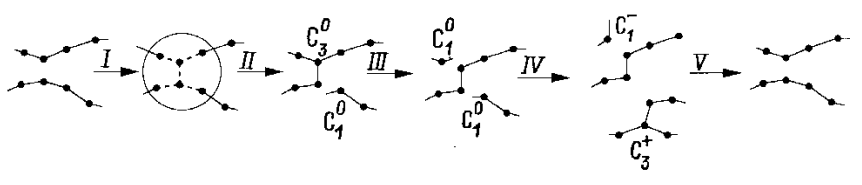

Figure 5. Structural change taking place in $a$-Se under photoexcitation and during the relaxation process. See text for details.

are formed (step I). Lattice relaxation and bond rupture leads to creation of neutral VAPs (step II) and appearance of the ESR signal. Three-fold coordinated defects are unstable and decay (step III) into singly coordinated defects (dangling bonds). At higher temperatures, pairs of neutral dangling bonds form charged VAPs (step IV) which is accompanied by disappearence of the ESR signal. Annealing at room temperature leads to recovery of the initial structure (step V).

Our main result, namely, the observed formation of additional (interchain) bonds in the photo-excited state is in sharp contrast to previous models $[1,18,35]$ whose essence is summarised in the review [1] as follows: "One major aspect of light-induced changes in short-range order is breaking of existing and formation of new bonds" ([1], p. 124) and "new bonds are formed in place of those broken" ([35], p. 80). Our results show that the realistic sequence of the processes is exactly the opposite: first dynamical interchain bonds are formed and the bond breaking follows. The crucial role in this process belongs to LP-electrons whose presence allows for the formation of such dynamic bonds. The obtained data make it clear why reversibly photostructural change only take place in amorphous chalcogenides and not in $a$-As or $a-\mathrm{Si}: \mathrm{H}$ - the last two do not possess LP-electrons and cannot undergo the bond switching that easily.

Irradiation of amorphous chalcogenides with linearly polarized light results in the formation of dynamic bonds in a selected direction finally resulting in redistribution of bonding and non-bonding electrons which manifests itself as photo-induced anisotropy [36,37].

\subsection{Photo-induced processes in binary chalcogenides}

In order to check whether the mechanism active in $a$-Se is also valid for more complex glasses we undertook studies of reversible photostructural changes in a binary glass $\mathrm{As}_{2} \mathrm{Se}_{3}$. We have found that in the binary glass as well the coordination of selenium species increases reversibly in the photoexcited state [24]. As well as in $a$-Se, irradiation of binary glasses results in an appearence of the ESR signal [34] which is annealed out at around room temperature. Secondary irradiation of the films at low temperature is characterised by faster kinetics [34]. Annealing at temperatures above room temperature leads to recovery of the initial parameters [1-3].

One can thus see that all set of phenomena is essentially the same for $a$-Se and complex glasses and we thus conclude that the underlying mechanism is also the same. It consists in bond switching via the formation of dynamic bonds due to photo-excitation of LP-electrons of chalcogen atoms. The process in binary chalcogenides is more complicated since, in addition to coordination defects, also stoichiometric defects ("wrong bonds") can be induced in the material through the formation of dynamic interchalcogen bonds $[13,14,21]$.

\subsection{Relationship between the photostructural change and reversible photodarkening}

After the valence-alternation model was first suggested [31] it was argued that photo-induced VAPs were responsible for the reversible photodarkening [38]. Observed increase in the ESR built-up upon secondary irradiation of pre-irradiated and then annealed films [34] was considered as a further argument in favour of this hypothesis.

Later, however, it was argued [39] that temperature behaviours of the photo-induced ESR and photodarkening were quite different: ESR disappeared at temperatures quite a bit lower than the photodarkening. This result was the grounds for a conclusion that photo-induced ESR and photodarkening were not related. This consept became a consensus. We believe, however, that this conclusion is wrong and that earlier suggestion relating the photodarkening to the photo-induced creation of VAPs has been correct

One should realize that disappearence of ESR does not mean disappearence of defects. ESR is not related to the presence of defects as such but to unpaired spins localized at these defects. A much faster ESR built-up in the secondary irradiation cycle is a clear proof that defects have not disappeared after the disappearence of the ESR signal.

The faster component in the secondary irradiation process can be a measure of the concentration of the photo-induced charged defects. Fig. 6 shows the temperature dependencies of the concentration of charged VAPs calculated in this way (for more details see [33]) and of the photodarkening. The data are normalized in such a way that the first point (at $160 \mathrm{~K}$ ) for the dataset for the charged VAPs concentration coincides with the corresponding point for the photodarkening. Remarkable agreement between the two

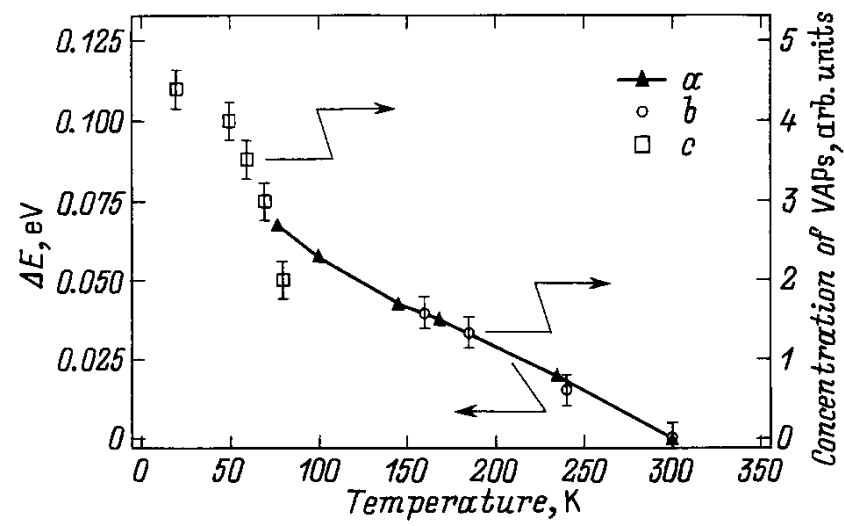

Figure 6. Temperature dependencies of the photodarkening $(a)$ (after [19]) and concentrations of neutral (c) and charged (b) VAPs. 
sets of data is clearly seen. Furthermore, we can estimate from Fig. 4 the concentration of neutral (ESR-active) VAPs and put on the same scale in the figure. One can see that the correspondence between the concentration of neutral VAPs and photodarkening is also quite good.

Detailed studies of the recovery of initial parameters in preirradiated $a$-Si [17] have revealed several steps of the process with the characteristic temperatures for the stages being 100 and $180 \mathrm{~K}$ which agrees with the characteristic temperatures of 90 and $160 \mathrm{~K}$ in the temperature behaviour of the photo-induced ESR. We can thus claim that reversible photodarkening is directly related to the presence of VAPs (either neutral or charged) in the material. Below we discuss why creation of VAPs results in photodarkening.

The top of the valence band in $a$-Se (and also in binary chalcogenides) is formed by LP-electrons. In the annealed state, LP-orbitals subtended at neighbouring atoms are oriented perpendicular to each other in order to minimize the energy. Any displacement from the equilibrium position will change mutial positions of atoms and orientation of LP-orbitals resulting in an increase in the energy of the system and causing broadening of the LP band which manifests itself as photodarkening. It is worthwile to notice that in all studied cahlcogenide glasses the reversible change in optical absorption is always photodarkening, although the irreversible component of such a change in non-equilibrated as-prepared films can be either darkening or bleaching depending on the sample composition and preparation conditions $[1,5]$.

We would like to stress here that photodarkening is not caused directly by the creation of photo-induced defects although they obviously make a certain contribution. More important, the photo-induced defects act as knots around which a structural change involving a much larger number of atoms takes place. It is this change in positions of the neighbouring atoms that is responsible for the reversible photodarkening. Similar approach was also used in refs. [40.41]. An electric field, introduced by charged defects present in high concentration, may also play a certain role for example through its effect on the excitonic absorption edge as discussed in [42].

\section{Conclusion}

Application of in-situ EXAFS, ESR and Raman scattering to studies of reversible photostructural changes on amorphous chalcogenides has allowed us to detect several stages of this process. The fundamental underlying process is the formation of dynamic interchain bonds in the photoexcited state due to excitaion of LP-electrons. Subsequent bond relaxation and rupture results in a metastable structural change.

Remarkable correlation between the photo-induced valence-alternation pairs and photodarkening has been shown and we claim that photo-creation of VAPs is the origin of the reversible photodarkening.

\section{Acknowledgements}

One of us (AVK) has started his research work in 1977 joining as a research student the laboratory headed by Professor B.T. Kolomiets and reversible photostructural changes in amorphous semiconductors have remained one of the main academic interests since that time. Live interest in this work expressed by Professor B.T. Kolomiets and his guidence have been the author's (AVK) priviledge for many years.

The results presented in this review would have been impossible without experimental assistance and numerous helfpul discussions with H. Oyanagi, M. Kondo and A. Roy whose participation in this work is gratefully acknowledged.

This work, partly supported by NEDO, was performed in the Joint Research Center for Atom Technology (JRCAT) under the joint research agreement between the National Institute for Advanced Interdisciplinary Research (NAIR) and the Angstrom Technology Partnership (ATP).

\section{References}

[1] G. Pfeifer, M.A. Paesler, S.C. Agarwal, J. Non-Cryst. Sol., 114, 130 (1989) and refs therein.

[2] Ke Tanaka. Rev. Sol. St. Sci., 4, 641 (1990).

[3] A. Kolobov, S.R. Elliott. Adv. Phys., 40, 625 (1991).

[4] Amorphous Silicon Technology-1996. MRS, vol. 420, ed. by M. Hack, E. Schiff, S. Wagner, R. Schropp and A. Matsuda (Pittsburg, Pennsylvania, 1996).

[5] K. Shimakawa, A.V. Kolobov, S.R. Elliott. Adv. Phys., 44, 475 (1995)

[6] J.S. Berkes, S.W. Ing, W.J. Hillegas. J. Appl. Phys., 42, 4908 (1971).

[7] S.A. Keneman. Appl. Phys. Lett., 19, 205 (1971).

[8] K. Tanaka. Appl. Phys. Lett., 26, 243 (1975).

[9] K. Tanaka, H. Hamanaka, S. Iijima. Proc. 7th Int. Conf. on Amorphous and Liquid Semiconductors, ed. by W.E. Spear (CICL, University of Edinburgh, 1977) p. 787.

[10] Ke Tanaka. J. Non-Cryst. Sol., 35-36, 1073 (1980).

[11] A.V. Kolobov, B.T. Kolomiets, O.V. Konstantinov, V.M. Lyubin. J. Non-Cryst. Sol., 45, 335 (1981).

[12] V.F. Krasnov, V.G. Remesnik. Avtometria, 4, 101 (1980).

[13] M. Frumar, A.P. Firth, A.E. Owen. Phil. Mag. B, 50, 463 (1984).

[14] C.Y. Yang, M.A. Paesler, D.E. Sayers. Phys. Rev. B, 36, 9160 (1987).

[15] S.B. Gurevich, N.N. Ilyashenko, B.T. Kolomiets, V.M. Lyubin, V.P. Shilo. Proc. 6th Int. Conf. on Amorphous and Liquid Semiconductors: Structure and Properties of Non-Crystalline Semiconductors, ed. by B.T. Kolomiets (Leningrad, 1995) p. 451.

[16] E. Mytilineou, P.C. Taylor, E.A. Davis. Sol. St. Commun., 35, 497 (1980).

[17] R. Chang. Mater. Res. Bull., 2, 145 (1967).

[18] V.L. Averyanov, A.V. Kolobov, B.T. Kolomiets, V.M. Lyubin. Phys. St. Sol. (a), 57, 81 (1980).

[19] Ke Tanaka, A. Odajima. Sol. St. Commun., 43, 961 (1982).

[20] A.V. Kolobov, H. Oyanagi, K. Tanaka, Ke Tanaka. Phys. Rev. B, 55, 726 (1997). 
[21] A.V. Kolobov, A. Roy, K. Tanaka. J. Non-Cryst. Sol. (to be published).

[22] A.V. Kolobov, M. Kondo, H. Oyanagi, R. Durny, A. Matsuda, K. Tanaka. Phys. Rev. B, 56, R485 (1997).

[23] E.A. Stern. Phys. Rev. B, 10, 3027 (1974).

[24] A.V. Kolobov, H. Oyanagi, K. Tanaka. Phys. Sol. St., 39, 64 (1997).

[25] H. Hisakuni, Ke Tanaka. Science, 270, 974 (1995).

[26] D. Hohl, R.O. Janes. Phys. Rev. B, 43, 3856 (1991).

[27] C. Bichara, A. Pellegatti, J.-P. Gaspard. Phys. Rev. B, 49, 6581 (1994).

[28] A. Axman, W. Gissler, A. Kollmar, T. Springer. Disc. Farad. Soc., 50, 74 (1970).

[29] N.F. Mott, E.A. Davis. Electronic Processes in NonCrystalline Materials (Oxford, Clarendon Press, 1979).

[30] M. Abkowitz. J. Chem. Phys., 46, 4537 (1967).

[31] M. Kastner, D. Adler, H. Fritzsche. Phys. Rev. Lett., 37, 1504 (1976).

[32] D. Vanderbilt, J.D. Joannopoulos. Sol. St. Commun., 35, 535 (1980).

[33] A.V. Kolobov, M. Kondo, H. Oyanagi, A. Matsuda, K. Tanaka. Phys. Rev. B (to be published).

[34] D.K. Biegelsen, R.A. Street. Phys. Rev. Lett., 44, 803 (1980).

[35] S.R. Elliott. J. Non-Cryst. Sol., 81, 71 (1986).

[36] A.V. Kolobov, V. Lyubin, T. Yasuda, K. Tanaka. Phys. Rev. B, 55, 23 (1997).

[37] A.V. Kolobov, V. Lyubin, T. Yasuda, M. Klebanov, K. Tanaka. Phys. Rev. B, 55, 8788 (1997).

[38] R.A. Street. Sol. St. Commun., 24, 363 (1977).

[39] J. Hautala, W.D. Ohlsen, P.C. Taylor. Phys. Rev. B, 38, 11048 (1988).

[40] A.V. Kolobov, G.J. Adriaenssens. Phil. Mag. B, 69, 21 (1994).

[41] P. Nagels, E. Sleeckx, R. Callaerts, L. Tichy. Sol. St. Commun., 94, 49 (1995).

[42] J.D. Dow, D. Redfield. Phys. Rev. B, 1, 3358 (1970).

Редактор Л.В. Шаронова 